\title{
COMPARISON OF BOILING, STEAMING, AIR FRYING, DEEP- FRYING, MICROWAVING AND OVEN-COOKING ON QUALITY CHARACTERISTICS OF keropok lekor (MALAYSIAN FISH SAUSAGE)
}

\author{
MARINA MOHD TAMSIR ${ }^{1}$, NURUL SHAZINI RAMLI ${ }^{2}$, MAHMUD AB RASHID NOR-KHAIZURA ${ }^{2}$, \\ RADHIAH SHUKRI ${ }^{1}$ and MOHAMMAD RASHEDI ISMAIL-FITRY ${ }^{1 *}$ \\ ${ }^{1}$ Department of Food Technology, Faculty of Food Science and Technology, \\ Universiti Putra Malaysia, 43400 UPM Serdang, Selangor, Malaysia \\ ${ }^{2}$ Department of Food Science, Faculty of Food Science and Technology, \\ Universiti Putra Malaysia, 43400 UPM Serdang, Selangor, Malaysia \\ *E-mail: ismailfitry@upm.edu.my
}

Accepted 2 September 2021, Published online 31 December 2021

\begin{abstract}
Keropok lekor $(K L)$ (Malaysian fish sausage) is usually boiled and then deep-fried for consumption. The combination of these cooking methods could produce greasy and fatty products thus negatively affect health in a long term. Other cooking methods could be better alternatives to the common methods. Therefore, this study aimed to investigate the effect of six cooking methods namely boiling, steaming, air frying, deep-frying, microwaving and oven-cooking on the $K L$ quality. The cooked samples were analysed for the moisture, fat, linear expansion, cooking yield, water holding capacity, colour, texture and sensory properties. Boiling showed the highest linear expansion $(3.02 \%)$, cooking yield (104.10\%) and water holding capacity $(41.04 \%)$. Meanwhile, deep frying significantly reduced $(p<0.05)$ the moisture $(39.83 \%)$ and increased the fat $(6.58 \%)$ contents, exhibited harder texture $(93.13 \mathrm{~N})$ and showed a higher yellowness value (8.23) compared to other cooking methods. No significant difference $(p>0.05)$ was found in other properties; however, panellists significantly $(p<0.05)$ preferred the crispiness and overall acceptability of the deep-fried followed by oven-cooked $K L$. Indeed, textures and colours had significant $(p<0.05)$ relationships with sensory properties. In conclusion, deep-frying and oven-cooking reduced the moisture content and increased the hardness of $K L$, which increased the panelists' preferences. However, deep-fried $K L$ had a higher fat content due to the cooking with oil; therefore, the oven-cooked $K L$ can be the healthier alternative.
\end{abstract}

Key words: Cooking methods, deep-fried, fish products, heat treatments, keropok lekor

\section{INTRODUCTION}

Manufacturing and production of formulated fish products have become progressively significant due to the varieties they can offer. It has been reported that world fish production for consumption increased tremendously from 67 per cent in the 1960s to 87 per cent in 2014. The figures are estimated to increase by 21 per cent in 2025 indicating the growing demand for fish products especially in developing countries (OECD-FAO, 2016). Keropok lekor is one example of the formulated fish product that resembles sausage in texture, hence known as Malaysian fish sausage. The keropok lekor is a very popular snack food in Malaysia and other Asian countries. It is made by

\footnotetext{
* To whom correspondence should be addressed.
}

mixing fish flesh, starch, flour, and seasonings such as salt, sugar and monosodium glutamate (MSG). The homogenous dough is rolled into a cylindrical shape, similar to a sausage, and is typically boiled to gelatinize the starch before being served or further deep-fried to obtain a more preferable texture and taste (Nor-Khaizura et al., 2009; Murad et al., 2017).

The formulations of keropok lekor (fish sausage) may vary, but the starch composition can be as high as 60 percent, which permits the fish sausage to expand during cooking and contributes to the crispy characteristics of the product. Lowvalue pelagic fishes like round scad (Decapterus maruadsi), round hering (Dussumieria hasellti) and sardine (Sardinella aurita) are often used in the production of the fish sausage (Kaewmanee et al., 2015). The fish species, the freshness of fish, the ratio 
of the primary substances and the cooking methods may influence the quality of the final product (Taewee, 2011).

Deep frying is a commonly used cooking method for fish sausage either after the rolling or boiling process (Nor-Khaizura et al., 2009). The fish sausage becomes crispy and crunchy outside but the inside texture is soft. This cooking method is very convenient and produced exclusive sensorial properties of fried food that is highly accepted by consumers (Bordin et al., 2013). During the deep frying process, the high temperature causes water to evaporate, oil is absorbed into the food and the aromatic compounds are released making the final product dominated by oil and the by-product of oil (Gertz et al., 2014; Sadgrove et al., 2015). As a result, deep-frying leads to an increase in fat content and a decrease in the shelf life of the product during storage due to the oxidation of fat (Kok et al., 2004).

Based on the fact that excessive consumption of fried products can lead to serious health risks such as obesity and hypertension (Qin et al., 2021), the food industry has put efforts into developing alternative methods to cook food products with low oil content but produce similar features of flavour, colour and texture. Many types of cooking methods are available, however to the best of our knowledge, there is limited information regarding the effect of different cooking methods of the keropok lekor on the colour and cooking characteristics as well as its sensory attributes or consumer expectations. Hence, the objectives of this study are to investigate the effects of different cooking methods namely boiling, steaming, air frying, deep-frying, microwaving and oven-cooking on physicochemical and sensory properties of keropok lekor.

\section{MATERIALS AND METHODS}

\section{Materials \\ Sardine (Sardinella aurita) and Mackerel (Rastrelliger kanagurta) were purchased from a local market located in Seri Kembangan, Selangor, Malaysia. Sago flour, tapioca starch, sugar, salt and MSG (Ajinomoto (Malaysia) Berhad, Malaysia) were obtained from a grocery store in Putrajaya, Malaysia.}

\section{Keropok lekor production}

The fresh fish was cleaned, washed and the flesh was separated using deboner (Ding-Han Machinery Co., Ltd., DH811-200, Taiwan). The flesh was put into a plastic bag, sealed and kept in a freezer at $-18^{\circ} \mathrm{C}$ before mixing with other ingredients to form the final dough. The dough was prepared with slight modifications according to Nur Liyana et al. (2019), which used fish flesh 50.2\%; sago flour
$17.5 \%$; tapioca starch $17.5 \%$; salt $1.3 \%$; sugar $0.8 \%$; MSG $0.2 \%$ and crushed ice $12.5 \%$. After mixing, the dough was rolled into a sausage-like shape weighed approximately $30 \mathrm{~g}$ each. The samples were packed in an air-tight polyethylene plastic bag and stored at $-18{ }^{\circ} \mathrm{C}$ for further cooking treatments and analyses.

\section{Cooking methods}

All samples were pre-boiled, which is a standard method in the processing of the keropok lekor. Water was heated in the pot until it reached the boiling point $\left(100^{\circ} \mathrm{C}\right)$. Then, samples were placed in the pot and boiled for $10 \mathrm{~min}$. Then, the boiled samples were divided into six groups, cooled down and dried at room temperature $\left(25-28^{\circ} \mathrm{C}\right)$. The first group was re-boiled for $10 \mathrm{~min}$ at $100{ }^{\circ} \mathrm{C}$. The second group was steamed at $100{ }^{\circ} \mathrm{C}$ for $1 \mathrm{~h}$ using a manual steamer. The third group was air-fried for $15 \mathrm{~min}$ at $150{ }^{\circ} \mathrm{C}$ using an air fryer (International Cooking Concepts Model ZM-AF400, Germany). The fourth group was deep fried in cooking oil using a domestic deep fryer (Stainless Steel Deep Fryer FDF 1035ss, Faber, Italy) for $6 \mathrm{~min}$ at $160{ }^{\circ} \mathrm{C}$. The fifth group was cooked in the microwave (Model R-360J(S), Sharp, Japan) for $2 \mathrm{~min}$ at a low power level (10P) which is equal to $80 \mathrm{~W}$. The sixth group was cooked for $20 \mathrm{~min}$ in the oven at $150{ }^{\circ} \mathrm{C}$.

\section{Moisture and fat analyses}

The moisture and fat contents of the samples were analyzed based on AOAC Method 934.01 and method 991.36, respectively (AOAC, 1990). Both analyses were conducted in triplicate.

\section{Linear expansion}

The length of the sample was measured before and after cooking using a vernier calliper and the linear expansion of the sample (\%) was calculated using the following formula (Murad et al., 2017):

\section{Linear expansion $(\%)=(\mathrm{LA}-\mathrm{LB}) / \mathrm{LB} \times 100 \%$}

in which, LA = length after cooked and LB = length before cooked.

\section{Cooking yield}

The percentage of cooking yield was determined by calculating the weight differences of the samples before and after cooking (Turp, 2016). The cooked samples were cooled to room temperature $\left(25-28^{\circ} \mathrm{C}\right)$ for $10 \mathrm{~min}$ and were reweighed to calculate the cooking yield using the following formula:

Cooking yield $(\%)=(\mathrm{WA} / \mathrm{WB}) \times 100 \%$

in which $\mathrm{WA}=$ weight after cooked and $\mathrm{WB}=$ weight before cooking 


\section{Water holding capacity}

The water holding capacity (WHC) was determined based on the method of Murad et al. (2017) with slight modifications. The samples (5 g) were blended and homogenized with $10 \mathrm{~mL}$ of distilled water. After centrifugation for $10 \mathrm{~min}$ at 2000 r.p.m., the supernatant was collected and calculated by using the formula below:

Water Holding Capacity $(\%)=(\mathrm{VB}-\mathrm{VA}) / \mathrm{WS} \times$ $100 \%$

where, $\mathrm{VB}=$ volume of water before centrifuged, VA $=$ volume of water after centrifuged, and WS = weight of the sample

\section{Colour analysis}

The colour of the cooked samples was measured using Konica Minolta portable colourimeter (CR-400, Konica Minolta, Japan $)$ for its lightness $(0=$ black, $100=$ white $)$, redness $\left(-a^{*}=\right.$ greenness, $+a^{*}=$ redness $)$ and yellowness $\left(-b^{*}=\right.$ blueness, $+b^{*}=$ yellowness $)$ ( $L^{*}, a^{*}$, and $b^{*}$ values, respectively).

\section{Texture profile analysis}

Texture profile analysis was carried out as described by Kaewmanee et al. (2015) with some modifications using a texture analyzer (TA-XTPlus, Micro Stable Systems, UK). The samples were compressed by a probe with a blade (HDP/BSK), using pre-test speed and post-test speed of 10 $\mathrm{mm} \mathrm{s} \mathrm{s}^{-1}$, test speed of $5 \mathrm{~mm} \mathrm{~s}^{-1}$ and distance of $10 \mathrm{~mm}$. The characteristics that had been analysed were hardness, cohesiveness, springiness, chewiness, and resilience.

\section{Sensory evaluation}

Sensory analysis was carried out based on Nur Liyana et al. (2019) with several modifications. Thirty consumer panellists received six random samples from the mid-section of fish sausages $(2 \mathrm{~cm} \times 2 \mathrm{~cm} \times$ $2 \mathrm{~cm}$ ) with questionnaires and instructions for the evaluation of samples using a hedonic scaling with a 9 -point scale $(1=$ dislike extremely, $9=$ like extremely). Sensorial analyses were conducted in a laboratory with individual booths located in the tasting room, which was air-conditioned and free of disturbing factors. The sensory attributes that had been tested include appearance, colour, texture, crispiness, taste, oiliness and overall acceptability.

\section{Statistical analysis}

Statistical analyses were conducted using Minitab statistical software (Minitab 19, Minitab Inc, Pennsylvania). The analysis of variance (ANOVA) was used to compare the data. Tukey's test was performed at confidence limits of 95\% $(P<0.05)$. Pearson correlation was also carried out to determine the correlation between selected parameters. All analyses were carried out in triplicate.

\section{RESULTS AND DISCUSSION}

\section{Moisture content, fat content, linear expansion, cooking yield and WHC}

Table 1 shows the moisture content, fat content, linear expansion, cooking yield and WHC of the keropok lekor obtained using different cooking methods. The moisture contents of air-fried, deep-fried and oven-cooked fish sausages were significantly reduced $(p<0.05)$ compared to other cooking methods. The deep-fried samples had the lowest moisture content $(39.83 \%)$ while the steamed and boiled samples displayed the highest value (55.78\% and 55.07\%, respectively). Steaming and boiling processes allowed the absorption of water into fish sausages resulted in increased moisture content. These results reflect the finding of Choi et al. (2016) who found that steaming of chicken steak produced higher moisture content than other cooking treatments.

The deep-frying method resulted in a significantly higher $(p>0.05)$ fat content $(6.58 \%)$ compared to other cooking methods $(1.59 \%-2.76 \%)$. Indeed, deep frying was the only method that used oil as a medium of cooking. Kassama and Ngadi (2016) showed a strong linear relationship between oil uptake and moisture loss during deep-frying of chicken breast indicated that deep-frying decreases the moisture content while increasing the fat content of the food. Similarly, García-Arias et al. (2003) suggested that the reduction of moisture content during frying contribute to an elevated level of fat in the cooked fish fillet. The result was likely caused by the penetration and absorption of oil into the sample during frying (Weber et al., 2008) after water is partially lost by evaporation (Saguy \& Dana, 2003). However, other factors such as the rate of change in food temperature and the temperature of the cooking method (higher in deep frying than in steaming) may influence the results (HassabAlla et al., 2009).

The boiled sample showed a significant increase $(p<0.05)$ of linear expansion compared to the air-fried sample. The steamed sample also had a positive expansion although not significantly different $(p>0.05)$ compared to others. Meanwhile, other cooking methods reduced the linear expansion of the samples. The positive values obtained by boiled and steamed samples were probably affected by the involvement of water uptake during the cooking process. Water is absorbed into the fish sausage during boiling, while during steaming, water vapour is entrapped inside the fish sausage and expanded. 
Other cooking methods such as air frying, deepfrying, microwaving, and oven-cooking showed negative values due to the shrinkage of the fish sausage. Taewee (2011) stated that starch is the key ingredient that causes the expansion and crispness of the snack. The finding is consistent with Huda et al. (2010) who reported that inadequate water during the baking process led to incomplete gelatinization of starch. It can thus be suggested that cooking fish sausage using air frying, deep-frying, microwaving, and oven-cooking methods reduced the starch gelatinization, hence decreased the expansion of the fish sausage.

The boiled and steamed samples resulted in significantly higher cooking yields $(p<0.05)$, while deep-fried and oven-cooked samples had the lowest significant $(p<0.05)$ cooking yield. This result can be reflected in the moisture content of the samples, in which water absorbed by the boiled and steamed samples increased the yield as compared to water removal for deep-fried and oven-cooked samples. A study by Trevisan et al. (2016) suggested that the moisture loss during frying, baking, and grilling decreased the cooking yield. Similarly, Das et al. (2013) proposed that the difference in cooking yield of food products is due to the variation in their moisture retention characteristics.

The highest WHC $(p<0.05)$ were shown in both boiled and steamed samples compared to other samples except for the microwaved samples. The WHC values obtained were significantly $(p<0.05)$ lower from deep-fried, oven-cooked and air fried samples. According to Suleman et al. (2020), the loss of water that occurs during frying leads to the aggregation of the protein. This aggregation causes the proteins to lose their three-dimensional structure, thus contribute to the loss of WHC. In addition, the contraction of collagen and denaturation of $\alpha$-actin and myosin of the samples could reduce WHC (Trevisan et al., 2016).

\section{Colour and texture profiles}

Colour and texture profiles of the keropok lekor treated with different cooking methods are shown in Table 2, while the appearances of the keropok lekor are displayed in Figure 1. Microwaved samples had significantly higher $L^{*}$ values demonstrating lighter colour than air-fried samples $(p<0.05)$. In contrast, no significant differences $(p>0.05)$ in $L^{*}$ values of the boiled, steamed, deep-fried and oven-cooked samples. The $L^{*}$ values depend on the cooking temperature which resulted in the pigment degradation on the outer layer of foods (Huda et al., 2011). Both deep frying and oven-cooking methods significantly increased $(p<0.05)$ the redness $\left(a^{*}\right)$ of the samples compared to other cooking methods. There was no significant difference $(p>0.05)$ of $a^{*}$ values between boiling, steaming, air frying and microwaving methods. About the yellowness parameter $\left(b^{*}\right)$, deep frying showed the highest value compared to the other cooking methods. Krokida et al. (2001) reported that during frying, heat and mass transfer phenomena take place, thus cause physicochemical changes, which affect the colour of the fried products.

The data obtained in the present study was consistent with the visual observation that the deepfried keropok lekor appeared golden yellow, thus significantly increase the yellow hue of keropok lekor. This result was similar to a study reported by Huda et al. (2001) who found that fried products had desirable homogenous yellow colour with higher $b^{*}$ values. In addition, several reactions occur during the heating of fish and meat including denaturation of protein and browning reaction that subsequently affect the colour and taste development of the food (HassabAlla et al., 2009; Das et al., 2013). A previous study indicated that Malaysian commercial fish sausages had variable colour characteristics range from 58.73-79.56 for $L^{*}$ value, $0.58-17.43$ for $a^{*}$ value and 12.69-22.96 for $b^{*}$ value (Huda et al., 2012). The present study, however, showed each cooking method presented different colour. The technique, medium of cooking, the composition of ingredients and condition of frying oil can also influence the formation of colour in food (Kulp et al., 2016).

The deep-fried sample showed the highest significant hardness $(p<0.05)$ among all the samples, followed by the oven-cooked samples $(p<0.05)$. The air-fried and microwaved samples had the medium significant $(p<0.05)$ hardness level, while the steamed and boiled samples were the least significant hard $(p<0.05)$ compared to other samples. The observed increase in hardness for the deep-fried and ovencooked samples could be attributed to crust formation that hardens the food surface as the frying temperature and the rate of heat transfer increases (Das et al., 2013). The hardness of muscle foods product is strongly influenced by the structural changes of their protein fractions due to the thermal, mechanical and ingredients used (Acosta et al., 2021). Besides, the lowest hardness in steamed and boiled samples could be due to weakened muscle structure and the leaching out of soluble protein as both cooking methods involved water (Li et al., 2017). Interestingly, the air fried sample had the highest significant $(p<0.05)$ chewiness level. This was followed by the boiled, steamed, oven-cooked, microwaved and deep-fried samples, although all these samples had no significant different changes $(p>0.05)$. No significant changes $(p>0.05)$ for the samples' cohesiveness, springiness, and resilience were observed among all the cooking methods. 


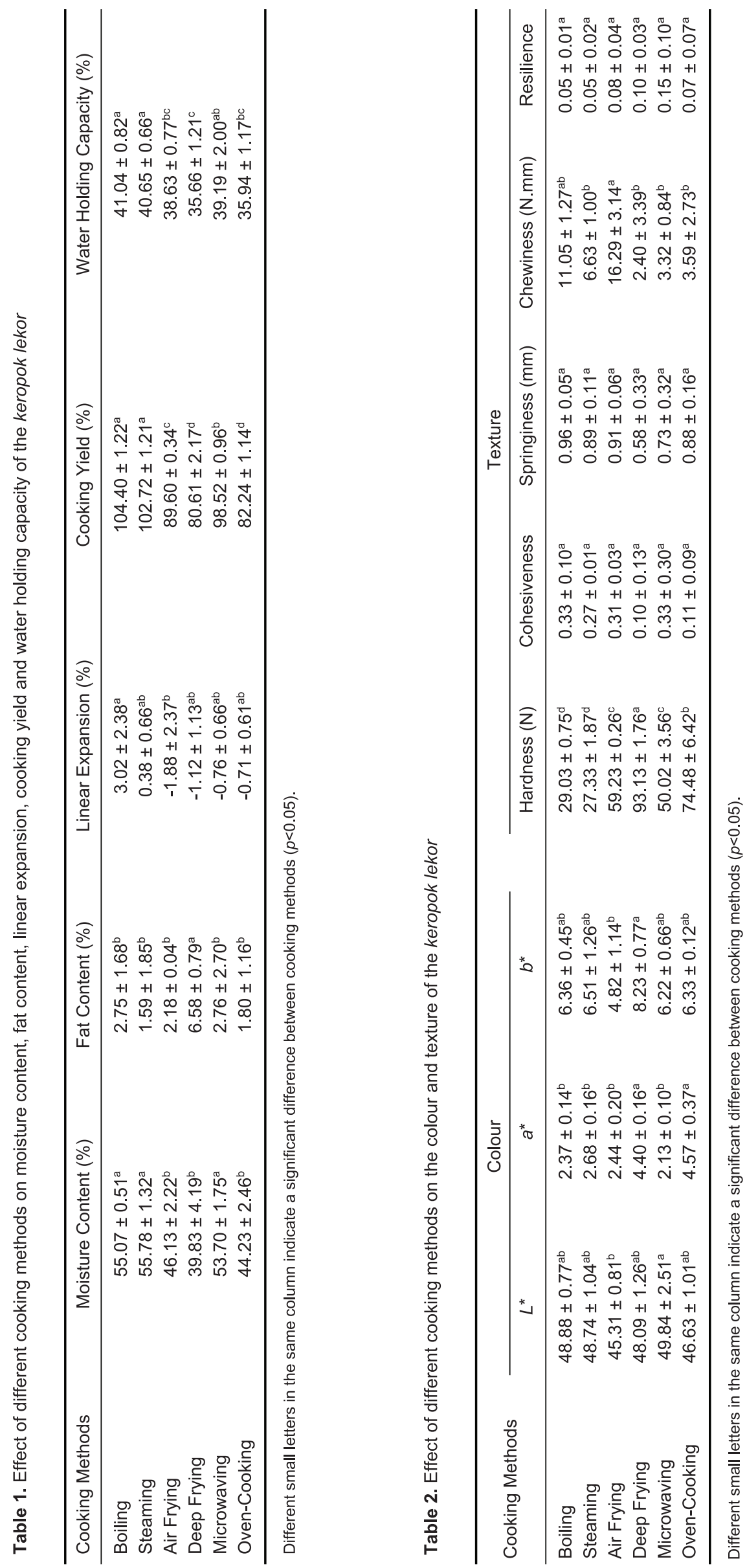




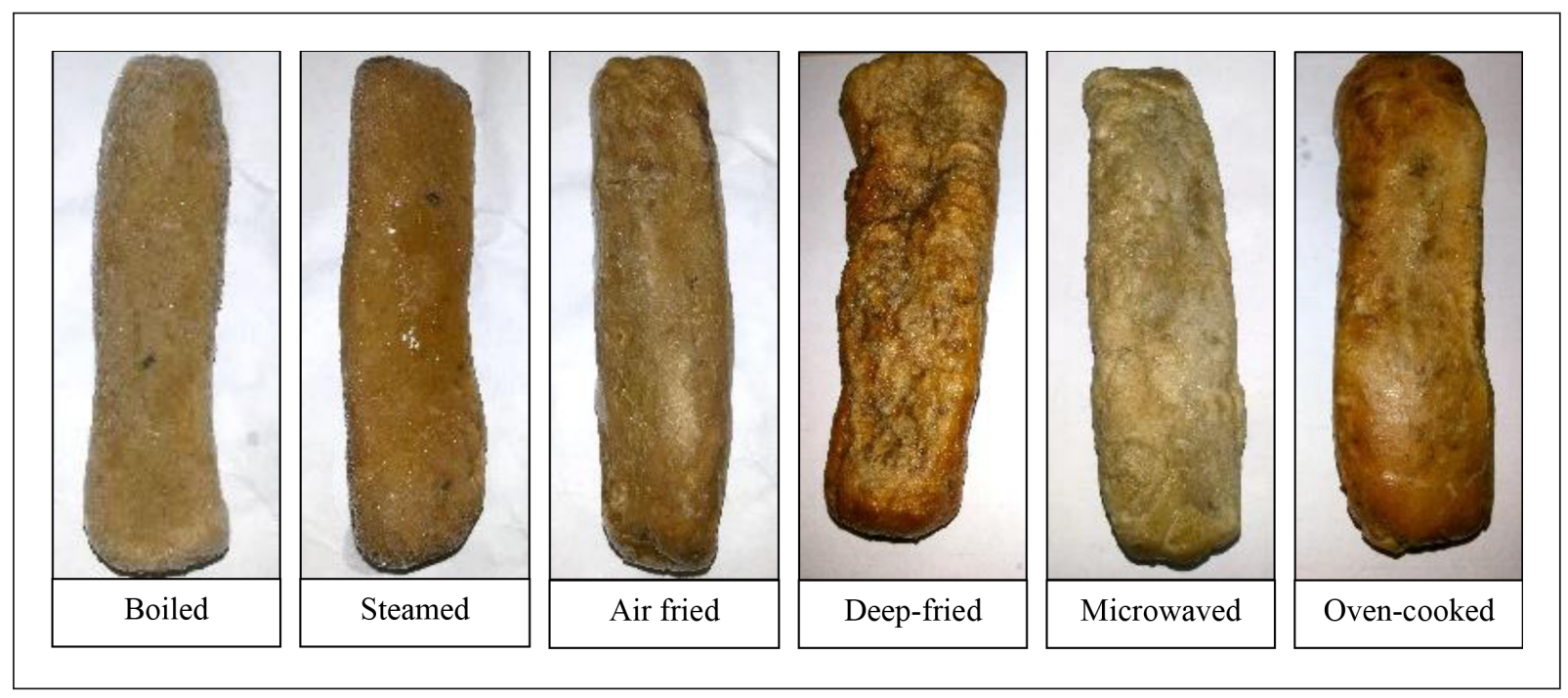

Fig. 1. Keropok lekor (Malaysian fish sausage) cooked using different methods.

\section{Sensory evaluation}

The result of the sensory evaluation of keropok lekor is presented in Table 3. Deep-fried keropok lekor consistently showed the highest value for all attributes. However, there were no significant differences in appearance, texture, and oiliness amongst all samples $(p>0.05)$. On the other hand, the crispiness and taste scores of deep-fried and ovencooked keropok lekor were significantly higher compared to other cooking methods $(p<0.05)$. These results are likely to be related to higher retention of natural juice and enhanced release of flavour compounds during deep frying and oven-cooking (Das et al., 2013). Furthermore, the keropok lekor will be immediately deep fry after rolling resulted in better taste than of the other cooking methods. The highest colour score of the deep-fried sample indicated that the panellists liked its colour. This could be due to caramelizing effect during deepfrying that produced uniform golden yellow colour. For overall acceptability, the panellists rated deepfrying as the cooking method that they like the most. The usage of oil during the deep-frying process increases the fat content of food products and people generally prefer the taste and texture of the fried foods due to the presence of fat that enhances the mouthfeel sensation (Kupiroviè et al., 2017). The results of the texture profile analysis were following those found by Giovanelli (2017) who observed better taste and texture of fish sausage, with softly cooked inside and crunchy outside when the deepfrying method was used.

\section{Relationship between the measured parameters}

Table 4 shows the relationship between the parameters with significant differences resulted from
Table 1, 2 and 3. The purpose was to determine the parameters and cooking methods that influenced most of the keropok lekor quality. Positive significant correlations $(p<0.05)$ were determined between the cooking yield and WHC against the moisture, and between the WHC against the cooking yield, indicating the final weight of cooked keropok lekor was influenced by the capability of retaining the water during the heat treatments. The hardness, crispiness, taste and overall acceptability had a negative significant correlation $(p<0.05)$ against the moisture, cooking yield and WHC. These results showed that cooking methods reduced the water content in keropok lekor, thus, created harder and crispier textures, which improved the preference among panellists. Interestingly, the colour, crispiness, texture, and overall acceptability were positively significant correlated $(p<0.05)$ against the redness $\left(a^{*}\right)$ values. The colour was a major factor for panellists especially the redness, which influenced their preference over other parameters. The crispiness, texture, and overall acceptability had positive significant correlations $(p<0.05)$ against the hardness and crispiness, indicating preference of the panellists towards crispier compared to soft keropok lekor. Panellists were also considered taste as the factor that influenced their overall acceptability, which was shown with positive significant correlations $(p<0.05)$ between these two parameters. To conclude, although the moisture content is important in determining the final weight of keropok lekor, the crispier and harder texture of keropok lekor with less water and redder colour was more preferred. 

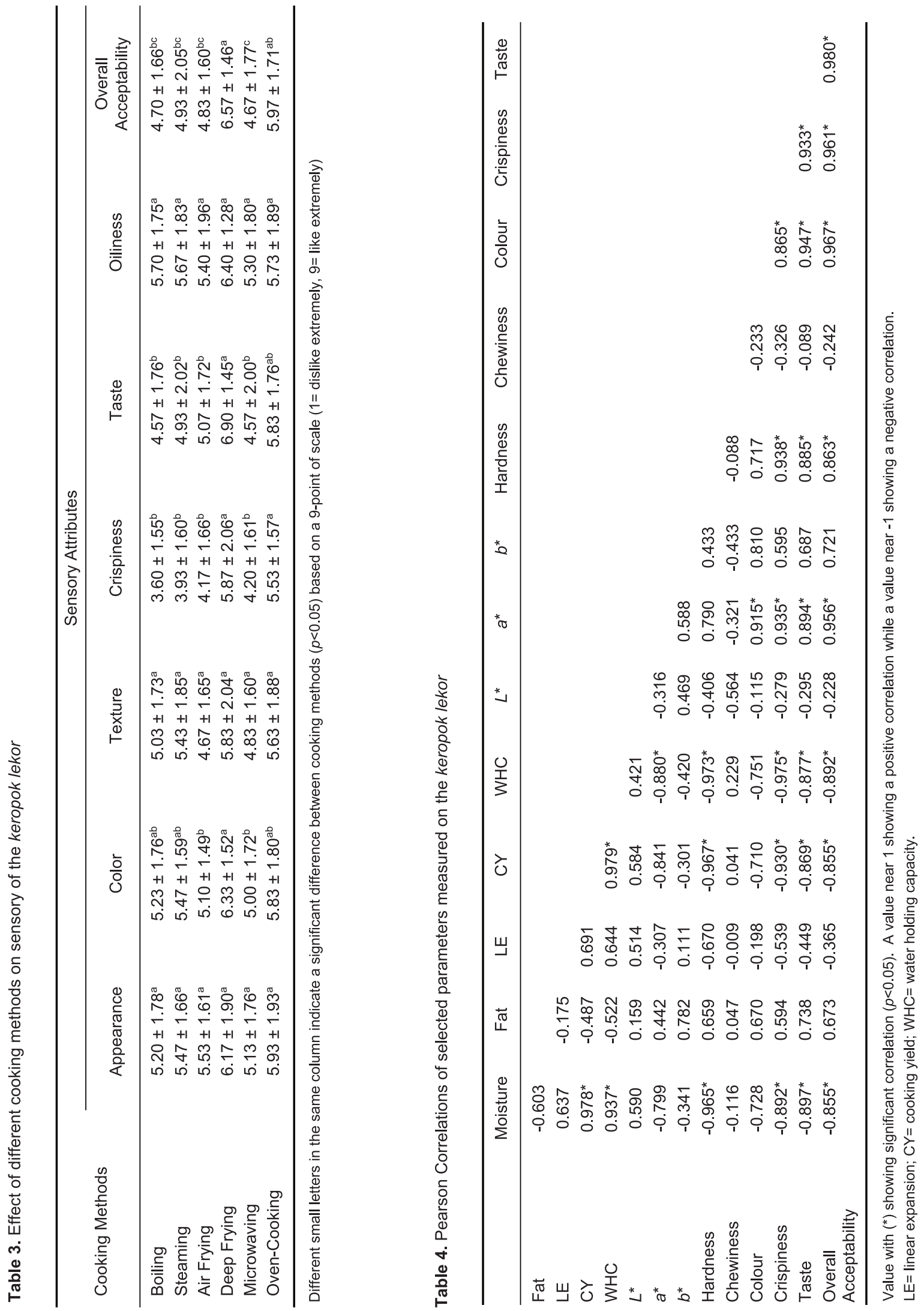


\section{CONCLUSION}

The different cooking methods (boiling, steaming, air frying, deep-frying, microwaving, and oven-cooking) had different effects on the physicochemical and sensory properties of the keropok lekor. Higher moisture content, cooking yield and water holding capacity were obtained by the boiled and steamed keropok lekor. In contrast, despite the highest fat content and hardest texture, deep-fried keropok lekor was the most preferred by the panellists influenced by the crispiness level. Oven-cooked keropok lekor had almost similar characteristics as the deep-fried keropok lekor but with lower fat content. Therefore, oven cooking can be used as an alternative to deep-frying to cook keropok lekor for a healthier option.

\section{REFERENCES}

Acosta, E.V., Ospina-E, J.C., Muñoz, D.A. \& Alvarez, H. 2021. Towards a phenomenological based model for predicting the hardness of a processed meat product. Journal of Food Science and Technology, 58(2): 701-709.

AOAC 1990. Official methods of analysis of AOAC International, $15^{\text {th }} \mathrm{Ed}$. AOAC International, Md., USA.

Bordin, K., Tomihe Kunitake, M., Kazue Aracava, K. \& Silvia Favaro Trindade, C. 2013. Changes in food caused by deep fat frying - A review. Archivos Latinoamericanos de Nutricion, 63(1): 5-13.

Choi, Y.S., Hwang, K.E., Jeong, T.J., Kim, Y.B., Jeon, K.H., Kim, E.M., Sung, J.M., Kim, H.W. \& Kim, C.J. 2016. Comparative study on the effects of boiling, steaming, grilling, microwaving and superheated steaming on quality characteristics of marinated chicken steak. Korean Journal for Food Science of Animal Resources, 36(1): 1-7.

Das, R., Pawar, D.P. \& Modi, V.K. 2013. Quality characteristics of battered and fried chicken: Comparison of pressure frying and conventional frying. Journal of Food Science and Technology, 50(2): 284-292.

García-Arias, M.T., Álvarez Pontes, E., GarcíaLinares, M.C., García Fernández, M.C. \& SánchezMuniz, F.J. 2003. Cooking-freezing reheating (CFR) of sardine (Sardina pilchardus) fillets. Effect of different cooking and reheating procedures on the proximate and fatty acid composition. Food Chemistry, 83(3): 349-356.

Gertz, C. 2014. Fundamentals of the frying process. European Journal of Lipid Science and Technology, 116(6): 669-674.
Giovanelli, G., Torri, L., Sinelli, N. \& Buratti, S. 2017. Comparative study of physico-chemical and sensory characteristics of French fries prepared from frozen potatoes using different cooking systems. European Food Research and Technology, 243(9): 1619-1631.

HassabAlla, A.Z., Mohamed, G.F., Ibrahim, H.M. \& AbdElMageed, M.A. 2009. Frozen cooked catfish burger: Effect of different cooking methods and storage on its quality. Global Veterinaria, 3(3): 216-226.

Huda, N., Alistair, T.L.J., Lim, H.W. \& Nopianti, R. 2012. Some quality characteristics of Malaysian commercial fish sausage. Pakistan Journal of Nutrition, 11(8): 700-705.

Kaewmanee, T., Karrila, T.T. \& Benjakul, S. 2015. Effects of fish species on the characteristics of fish cracker. International Food Research Journal, 22(5): 2078-2087.

Kassama, L.S. \& Ngadi, M. 2016. Relationship between oil uptake and moisture loss during deep fat frying of deboned chicken breast meat. Advances in Chemical Engineering and Science, 6(4): 324-334.

Kyaw, Z.Y. 2004. Protein-Hydrocolloids Interaction In "Keropok Lekor" (Ph.D). Universiti Putra Malaysia, Malaysia.

Kok, T.N., Yeap, S.E., Lee, W.P., Lee, H.K. \& Arig, G.T. 2004. Innovative techniques for traditional dried fish products. Developments in Food Science, 42: 397-406.

Krokida, M.K., Oreopoulou, V., Maroulis, Z.B. \& Marinos-Kouris, D. 2001. Colour changes during deep fat frying. Journal of Food Engineering, 48(3): 219-225.

Kulp, K., Loewe, R., Lorenz, K. \& Gelroth, J. 2016. Batters and Breadings in Food Processing. $2^{\text {nd }}$ Ed, Academic Press, Kansas, 337 pp.

Kupiroviř, U.P., Elmadfa, I., Juillerat, M.A. \& Raspor, P. 2017. Effect of saliva on physical food properties in fat texture perception. Critical Reviews in Food Science and Nutrition, 57(6): 1061-1077.

Li, C.H., Bland, J.M. \& Bechtel, P.J. 2017. Effect of precooking and polyphosphate treatment on the quality of microwave cooked catfish fillets. Food Science \& Nutrition, 5(3): 812-819.

Murad, N.S., Ismail, M.A., Nor-Khaizura, M.A.R. \& Ismail-Fitry, M.R. 2017. The effect of mixing time and mixing sequence during processing on the physicochemical and sensory properties of keropok lekor. Journal of Science and Technology, 9(4): 88-95. 
Nor-Khaizura, M.A.R., Zaiton, H., Jamilah, B., Rusul, R.A.G. \& Ismail-Fitry, M.R. 2009. Histamine and histamine-forming bacteria in Keropok lekor (Malaysian fish sausage) during processing. Food Science and Technology Research, 15(4): 395-402.

Nur Liyana, N., Nor-Khaizura, M.A.R. \& Ismail-Fitry, M.R. 2019. Effect of substituting tapioca starch with various high protein legume flours on the physicochemical and sensory properties of keropok lekor (Malaysian fish sausage). Food Research, 3(1): 40-48.

OECD/FAO 2016. "Fish and Seafood". In: OECDFAO Agricultural Outlook 2016-2025, OECD Publishing, Paris. pp. 113-115.

Qin, P., Liu, D., Wu, X., Zeng, Y., Sun, X., Zhang, Y., Li, Y., Wu, Y., Han, M., Qie, R. \& Huang, S. 2021. Fried-food consumption and risk of overweight/ obesity, type 2 diabetes mellitus, and hypertension in adults: a meta-analysis of observational studies. Critical Reviews in Food Science and Nutrition, 1-12.

Sadgrove, N. \& Jones, G. 2015. A contemporary introduction to essential oils: chemistry, bioactivity and prospects for Australian agriculture. Agriculture, 5(1): 48-102.
Saguy, S. \& Dana, D. 2003. Integrated approach to deep fat frying: Engineering, nutrition, health and consumer aspects. Journal of Food Engineering, 56(2-3): 143-152.

Suleman, R., Wang, Z., Aadil, R.M., Hui, T., Hopkins, D.L. \& Zhang, D. 2020. Effect of cooking on the nutritive quality, sensory properties and safety of lamb meat: Current challenges and future prospects. Meat Science: 167: 108172.

Taewee, T.K. 2011. Cracker "Keropok": a review on factors influencing expansion. International Food Research Journal, 18(3): 855-866.

Trevisan, A.J.B., de Almeida Lima, D., Sampaio, G.R., Soares, R.A.M. \& Bastos, D.H.M. 2016. Influence of home cooking conditions on Maillard reaction products in beef. Food Chemistry, 196: 161-169.

Turp, G.Y. 2016. Effects of four different cooking methods on some quality characteristics of low fat Inegol meatball enriched with flaxseed flour. Meat Science, 121: 40-46.

Weber, J., Bochi, V.C., Ribeiro, C.P., Victório, A.D.M. \& Emanuelli, T. 2008. Effect of different cooking methods on the oxidation, proximate and fatty acid composition of silver catfish (Rhamdia quelen) fillets. Food Chemistry, 106(1): 140-146. 
